Reachability in one-dimensional controlled polynomial dynamical systems

Korovina, Margarita and Vorobjov, Nicolai

2011

MIMS EPrint: 2011.47

Manchester Institute for Mathematical Sciences

School of Mathematics

The University of Manchester

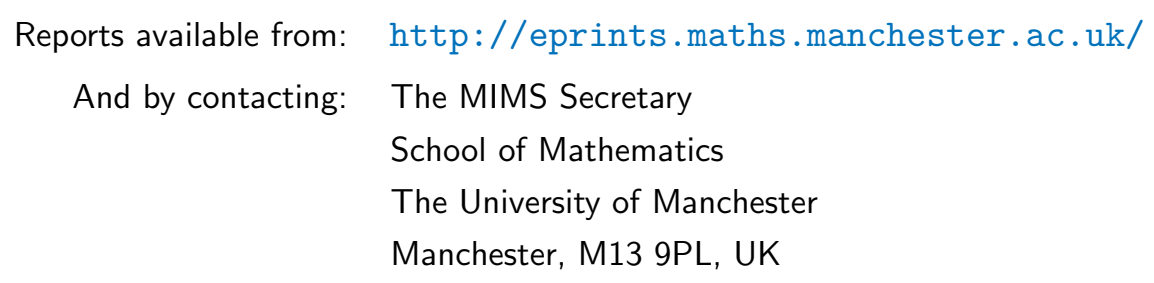

ISSN 1749-9097 


\title{
Reachability in one-dimensional controlled polynomial dynamical systems *
}

\author{
Margarita Korovina $^{1}$ and Nicolai Vorobjov ${ }^{2}$ \\ 1 The University of Manchester and IIS SB RAS Novosibirsk, \\ Margarita.Korovina@manchester.ac.uk, \\ 2 University of Bath, UK \\ nnv@cs.bath.ac.uk
}

\begin{abstract}
In this paper we address the reachability problem in controlled o-minimal dynamical systems. This problem can be formulated as follows. Given a controlled o-minimal dynamical system and a target set, starting at an initial point, is there a finite choice of time points and control parameters applied at these points such that the target set is reachable. We prove the existence of a finite control stategy and construct an algorithm which provides finite control strategies for one-dimensional controlled polynomial dynamical systems. We also provide a complexity result which shows upper bounds on the numbers of switches in finite control strategies.
\end{abstract}

\section{Introduction}

A fundamental problem in the design of biological, chemical or physical processes is to automatically synthesise models from performance specifications. In general, by practical and theoretical reasons, it is highly nontrivial to achieve. However, in some cases, given by partial designs, it could be possible to automatically complete modelling in order to get desired properties.

In this paper we consider synthesis of finite control strategies to meet reachability and time requirements for partial designs given by controlled polynomial dynamical systems (CPDS).

A controlled polynomial dynamical system is defined by a polynomial depending on control parameters. The choice of a parameter determines a certain motion. In general case, in order to archieve reachability or time requirements it is necessary to switch between motions corresponding to various control parameters in certain moments of time. We focus on the following problem.

Problem of finite control synthesis. For a partial design, given by CPDS, determine whether there exists finite sequences of time moments and control parameters that lead the system from an initial state to a desired state. If the answer is positive then automatically synthesise a finite control strategy.

\footnotetext{
* This research was partially supported by EPSRC grant EP/E050441/1, DFG-RFBR
} 09-01-91334-NNIO-a 
In the general class of o-minimal dynamical systems this problem does not have a postive solution as demonstrated in $[1,2,6]$. Indeed, it has been shown that the reachability problem is already undecidable for three-dimensional piecewise constant derivative systems and two-dimensional o-minimal dynamical systems with non-determinism. Moreover, for one-dimensional o-minimal dynamical systems with non-determinism, the problem remains open. However, our investigations show that it is possible to find finite control strategies for certain broad classes of o-minimal dynamical systems. In this paper we consider the problem for one-dimensional controlled polynomial dynamical systems. The main contributions of the paper are the proof of the existence of a finite control stategy and an algorithm synthesising finite control strategies for one-dimensional controlled polynomial dynamical systems based on quantifier elimination for real closed fields and real algebraic geometry tools. We also provide a complexity result which shows upper bounds on the numbers of switches in finite control strategies.

The paper is organised as follows. In Section 2 we define the problem of finite control strategy synthesis for controlled polynomial dynamical systems. Section 3 contains essential relative properties of the integral curves of a given dynamics. In Section 4 we construct an algorithm synthesising finite control strategies and show upper bounds on the number of switches. We conclude with future and related work.

\section{Problem Description}

In this section we formalise the problem of finite control strategy synthesis for controlled polynomial dynamical systems. Let

$$
\begin{gathered}
\gamma: Y \times T \rightarrow X \\
(y, t) \mapsto x,
\end{gathered}
$$

be a polynomial function, where $Y \subseteq \mathbb{R}^{m}, T \subseteq \mathbb{R}$ and $X \subseteq \mathbb{R}^{n}$. We consider $\gamma$ as a controlled dynamical system with control parameters from $Y$. For every fixed control value $y_{0} \in Y$ the function $\gamma\left(y_{0}, t\right): T \rightarrow X$ describes the motion of a point in the state space $X$. In what follows we will use the notation $x^{y_{0}}(t)$ for $\gamma\left(y_{0}, t\right)$, and call the function $x^{y_{0}}(t)$ integral curve.

We are considering the following problem of reachability: given a point and a subset in $X$, decide whether or not the subset can be reached from the point using a combination of motions corresponding to various controls.

Definition 1. Let $t_{0}, t_{1}, \ldots, t_{k} \in T$ be moments of time and $y_{0}, y_{1}, \ldots, y_{k} \in Y$ be controls. Assume also that $x^{y_{j}}\left(t_{j+1}\right)=x^{y_{j+1}}\left(t_{j+1}\right)$ for every $j \in\{0, \ldots, k-1\}$. Then

$$
C=\bigcup_{0 \leq j \leq k-1}\left\{(t, x) \in T \times X \mid t \in\left[t_{j}, t_{j+1}\right], x=x^{y_{j}}(t)\right\}
$$

is called piecewise integral curve and the tuple $\left\langle\left(t_{0}, y_{0}\right), \ldots,\left(t_{k}, y_{k}\right)\right\rangle$ is called finite control strategy. 
Now we can reformulate the reachability problem formally as follows. Given a polynomial function $\gamma$, initial conditions $\left(t_{0}, x_{0}\right) \in T \times X$ and a definable target subset $\Omega \subset X$ decide whether there exists a piecewise integral curve $C$ reaching $\Omega$, i.e., such that $\Omega \cap x^{y_{k}}(t) \neq \emptyset$.

If the polynomial in $\gamma$ has integer coefficients while $Y, T$ and $X$ are "simple" enough (e.g., open intervals on the straight line) then the reachability problem can be considered as computational, and one can ask the natural questions about decidability and computational complexity. An interesting weaker question is to bound from above the number $k$ in a reaching piecewise integral curve $C$ in the case when an instance of the computational problem has the positive output.

Remark 1. It is worth noting that in this case, by quantifier elimination for the first order theory of the reals, the finite control strategy problem is semidecidable.

Remark 2. If we fix an upper bound on the number of switches $k$ then the finite control strategy problem become to be decidable.

Now we assume that $Y, T, X \subset \mathbb{R}$ are open intervals, and

$$
\Gamma:=\{(y, t, x) \mid x=\gamma(y, t)\} \subset Y \times T \times X \subset \mathbb{R}^{3}
$$

is the graph of the function $\gamma$. Denote by $\pi: \Gamma \rightarrow T \times X$ the projection map, and let $U$ be one of the connected open sets into which the set of all critical values of $\pi$ divides $\pi(\Gamma)$. Then all fibres $\pi^{-1}(x, t)$ for $(x, t) \in U$ are finite and have the same cardinality $\ell$. Moreover, by the implicit function theorem, $\pi^{-1}(U)$ is a union of $\ell$ smooth surfaces (graphs of smooth functions) $\Gamma^{1}, \ldots, \Gamma^{\ell}$ such that the restriction of $\pi$ on each $\Gamma^{i}$ is a diffeomorphism.

Observe that for any $\Gamma^{i}$ and $y_{1} \neq y_{2}$, the projections $x^{y_{1}}(t)$ and $x^{y_{2}}(t)$ of the two non- empty intersections $\Gamma^{i} \cap\left\{y=y_{1}\right\}$ and $\Gamma^{i} \cap\left\{y=y_{2}\right\}$ respectively on $T \times X$, have the empty intersection, $x^{y_{1}}(t) \cap x^{y_{2}}(t)=\emptyset$, because $\Gamma^{i} \cap\{y=$ $\left.y_{1}\right\}, \Gamma^{i} \cap\left\{y=y_{2}\right\}$ are two different level sets of a function. On the other hand, for $\Gamma^{i_{1}}, \Gamma^{i_{2}}$, where $i \neq j$, the projections $x^{y_{1}}(t), x^{y_{2}}(t)$ of non-empty intersections $\Gamma^{i_{1}} \cap\left\{y=y_{1}\right\}, \Gamma^{i_{2}} \cap\left\{y=y_{2}\right\}$, being smooth real algebraic curves may intersect, either by coinciding or at a finite number of points.

It follows, that in any piecewise integral curve $C$, restricted on $U$, any two subsequent integral curves $x^{y_{j}}(t), x^{y_{j+1}}(t)$ are projections on $T \times X$ of intersections $\Gamma^{i_{1}} \cap\left\{y=y_{j}\right\}, \Gamma^{i_{2}} \cap\left\{y=y_{j+1}\right\}$ with $i_{1} \neq i_{2}$. In particular, at any point $(x, t) \in U$ there is a finite number of possible choices of the control.

It seems unavoidable to consider, as a part of the general reachability problem, its following restriction. Given $\gamma$, an open connected subset $U$ of regular values of the projection $\pi$, initial conditions $\left(t_{0}, x_{0}\right) \in U$ and the target point $\omega \in X$ decide whether there exists a piecewise integral curve $C \subset U$ such that $\omega \in x^{y_{k}}(t)$. In this paper we consider, in essence, this restricted version of reachability.

Let $U$ be the square $(-1,1) \times(0,1)$ with coordinates $(t, x)$. Consider $\ell$ families $x_{\alpha}^{i}(t)$ of disjoint graphs of functions in $U$, where $i \in\{1, \ldots, \ell\}$ enumerates families and $\alpha \in \mathbb{R}$ parametrises continuously functions within the family. 


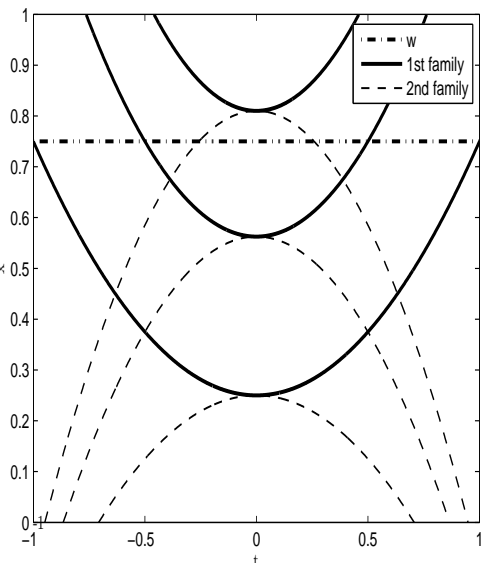

(a) Integral curves

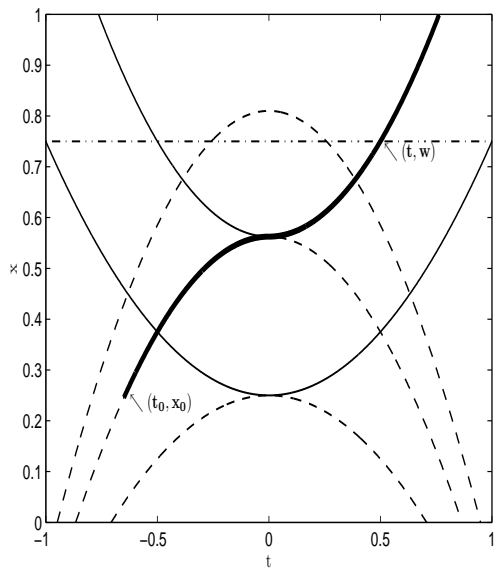

(b) A piescewise integral curve

Fig. 1: The pictures illustrate Example 1. In Picture 1a the first family of integral curves corresponds to $y \in(0,1)$, and the second family of integral curves correspons to $y \in(-1,0)$. The piescewise integral curve in Picture $1 \mathrm{~b}$ correspons to the strategy $<\left(t_{0}, y_{0}\right),\left(t_{1}, y_{1}\right)>=<\left(-\frac{3}{5},-\frac{3}{4}\right),\left(0, \frac{3}{4}\right)>$ which solves the reachability problem for $\left(t_{0}, x_{0}\right)=\left(-\frac{3}{5}, \frac{117}{400}\right)$ and $\Omega=\{x \mid 1>x \geq \omega\}$.

More precisely, there are $\ell$ surjective Nash functions $\Gamma^{i}: U \rightarrow \mathbb{R}, 1 \leq i \leq \ell$ (i.e., real analytic functions such that $P\left(t, x, \Gamma^{i}(t, x)\right)=0$ for some polynomial $P \in \mathbb{R}\left[z_{1}, z_{2}, z_{3}\right]$ and every $(t, x) \in U$, see [3]). Assume that for every $i$ and for every $\alpha \in \mathbb{R}$ the level set $x_{\alpha}^{i}(t):=\left\{(t, x) \in U \mid \Gamma^{i}(t, x)=\alpha\right\}$ is either empty or a graph of a smooth function on a subinterval of $(-1,1)$ of the $t$-axis. It follows that for each point $\left(t_{0}, x_{0}\right) \in U$ and every $i \in\{1, \ldots, \ell\}$ there is $\alpha \in \mathbb{R}$ such that $x_{\alpha}^{i}\left(t_{0}\right)=x_{0}$.

The aim is, having the initial point $\left(t_{0}, x_{0}\right) \in U$ to find the moments of time $t_{1}, \ldots, t_{k} \in(-1,1)$ and the corresponding controls $i_{1}, \ldots, i_{k}$ such that the piecewise integral curve

$$
C=\bigcup_{0 \leq j \leq k-1}\left\{(t, x) \in U \mid t \in\left[t_{j}, t_{j+1}\right], x=x_{j}^{i_{j}}(t)\right\}
$$

reaches the set $\Omega \subseteq X$. (Here the notation $x_{r}^{i}(t)$ is used for the integral curve belonging to the family $i$ and passing through the point $\left(x_{r}, t_{r}\right)$ under the control $i \in\{1, \ldots, \ell\}$.) Of course, if $x_{0} \in \Omega$ then the problem solves trivially.

Example 1. Let a polynomial function $\gamma:=y^{2}+y t^{2}$ be defined on $(-1,1) \times$ $(-1,1)$ and $\Omega=\{x \mid x \geq \omega\}$. The set of all critical values, $\left\{(t, x) \mid x=-t^{4} / 4\right\}$, of the projection map $\pi:\left\{(y, t, x) \in \mathbb{R}^{3} \mid x=y^{2}+y t^{2}\right\} \rightarrow \mathbb{R}^{2}$ lies outside $U$, and $\ell=2$. 
Let $\Gamma^{1}(U)=(0,1)$ and $\Gamma^{2}(U)=(-1,0)$. Observe that for every $y \in(0,1)$ (respectively, $y \in(-1,0)$ ) the integral curve $x_{y}^{1}(t)$ (respectively, $x_{y}^{2}(t)$ ) is a parabola having a minimum (respectively, maximum) at $t=0$.

Assume that $x_{0}<\omega$. It is easy to see that there is a piecewise integral curve reaching $\omega \in(0,1)$ if and only if the initial point $\left(t_{0}, x_{0}\right)$ satisfies either the conjunction of inequalities $x_{0} \geq \alpha^{2}-\alpha t_{0}^{2}, t_{0} \leq 0$ or of inequalities $x_{0} \geq$ $\alpha^{2}+\alpha t_{0}^{2}, t_{0} \geq 0$, where $\alpha:=(-1+\sqrt{1+4 \omega}) / 2$.

Suppose that $x_{0} \geq \alpha^{2}-\alpha t_{0}^{2}$ and $t_{0} \leq 0$, and let $\beta \geq \alpha$ be such that $x_{-\beta}^{2}(t)$ passes through $\left(t_{0}, x_{0}\right)$. If $\omega>\beta^{2}$ then the target $\omega$ is reached by the piecewise integral curve

$$
\left\{(t, x) \in U \mid t \in\left[t_{0}, 0\right], x=x_{-\beta}^{2}(t)\right\} \cup\left\{(t, x) \in U \mid t \in\left[0, t_{2}\right], x=x_{\beta}^{1}(t)\right\},
$$

where $x_{\beta}^{1}\left(t_{2}\right)=\omega$. If $\omega \leq \beta^{2}$ then $\omega$ is reached by the piecewise integral curve

$$
\left\{(t, x) \in U \mid t \in\left[t_{0}, t_{1}\right], x=x_{-\beta}^{2}(t),\right.
$$

where $x_{-\beta}^{2}\left(t_{1}\right)=\omega$.

Suppose that $x_{0} \geq \alpha^{2}+\alpha t_{0}^{2}$ and $t_{0} \geq 0$, and let $\delta \geq \alpha$ be such that $x_{\delta}^{1}(t)$ passes through $\left(t_{0}, x_{0}\right)$. If $\omega>\delta^{2}$ then $\omega$ is reached by

$$
\left\{(t, x) \in U \mid t \in\left[t_{0}, t_{1}\right], x=x_{\delta}^{1}(t)\right\},
$$

where $x_{\delta}^{1}\left(t_{1}\right)=\omega$. Otherwise, $x_{0}$ is already in $\Omega$.

Note that under any of these suppositions there may be other piecewise integral curves reaching the target. On the other hand, it is easy to prove that the described above motion is the fastest one.

\section{Relative properties of integral curves}

In this section we prove basic properties of integral curves of CPDS which we use later in the synthesis of finite control strategies.

Observe that any integral curve $x_{\alpha}^{i}(t)$, being a graph of a continuous function, divides $U$ into two disjoint subsets, $U_{+}(i, \alpha)$ which contains a point $\left(t^{\prime}, x^{\prime}\right) \in U$ such that $x^{\prime}>x_{\alpha}^{i}\left(t^{\prime}\right)$, and $U_{-}(i, \alpha)$ which contains a point $\left(t^{\prime \prime}, x^{\prime \prime}\right) \in U$ such that $x^{\prime \prime}<x_{\alpha}^{i}\left(t^{\prime \prime}\right)$.

Definition 2. 1. A point $\left(t^{\prime}, x^{\prime}\right) \in U$ is called above an integral curve $x_{\alpha}^{i}(t)$ if $\left(t^{\prime}, x^{\prime}\right) \in U_{+}(i, \alpha)$.

2. For two integral curves $x_{\alpha}^{i}(t)$ and $x_{\beta}^{i}(t)$ from the same family $i, x_{\beta}^{i}(t)$ is called above $x_{\alpha}^{i}(t)$ if $x_{\beta}^{i}(t) \subset U_{+}(i, \alpha)$.

3. For two integral curves $x_{\alpha}^{i}(t)$ and $x_{\beta}^{j}(t)$ from two different families $i \neq j$, $x_{\beta}^{j}(t)$ is called locally above $x_{\alpha}^{i}(t)$ at $\left(t_{0}, x_{0}\right) \in U$ if there is a neighbourhood $U_{0}$ of $\left(t_{0}, x_{0}\right)$ in $U$ such that $x_{\beta}^{i}(t) \cap U_{0} \subset U_{+}(i, \alpha) \cap U_{0}$.

4. We say that $\left(t_{0}, x_{0}\right)$ is a local minimum point of $x_{\beta}^{j}(t)$ relative to $x_{\alpha}^{i}(t)$ if $x_{\alpha}^{i}(t)$ and $x_{\beta}^{j}(t)$ are tangent at $\left(t_{0}, x_{0}\right)$, and $x_{\beta}^{j}(t)$ is locally above $x_{\alpha}^{i}(t)$. 
Lemma 1. Let $\left(t_{0}, x_{0}\right)$ be a local minimum point of $x_{\beta}^{j}(t)$ relative to $x_{\alpha}^{i}(t)$. There are no other local minimum points of $x_{\lambda}^{j}(t)$ relative to $x_{\alpha}^{i}(t)$ in a neighbourhood of $\left(t_{0}, x_{0}\right)$ on $x_{\alpha}^{i}(t)$ for any $\lambda$. In particular, there is a finite number of local minima relative to $x_{\alpha}^{i}(t)$.

Proof. If the claim is not true then there is a point $\left(t^{\prime}, x^{\prime}\right) \in x_{\alpha}^{i}(t)$ and an integral curve $x_{\lambda}^{j}(t)$, having a local minimum at $\left(t^{\prime}, x^{\prime}\right)$ relative to $x_{\alpha}^{i}(t)$, such that $x_{\beta}^{j}(t) \cap x_{\lambda}^{j}(t) \neq \emptyset$. This contradicts to the condition that integral curves in the same family do not intersect.

Definition 3. Let $G_{i, j}$ denote the set of all points $(t, x) \in U$ such that $(t, x)$ is a point of local minimum of $x_{\beta}^{j}(t)$ relative to $x_{\alpha}^{i}(t)$ for some $i, j$.

Corollary 1. 1. $G_{i, j}$ is a curve in $U$.

2. $G_{i, j} \cap G_{j, i}=\emptyset$.

Proof. (1) According to Lemma 1, for every fixed $\alpha$ there is a finite number of minima relative to $x_{\alpha}^{i}(t)$. Considering $\alpha$ as a parameter, we obtain a semialgebraic curve.

(2) Obvious from the definition of $G_{i, j}$.

Lemma 2. Each connected component of the curve $G_{i, j}$ is homeomorphic to the interval $(0,1)$ and is intersected by each curve $x_{\alpha}^{i}(t)$ at most once, and by each curve $x_{\beta}^{j}(t)$ at most once.

Proof. Suppose that a connected component $G$ of $G_{i, j}$ is intersected by a certain integral curve $x_{\alpha}^{i}(t)$ at more than one point. Let $A$ and $B$ be some two neighbouring among them. Then there will be curves $x_{\lambda}^{i}(t)$ in the family $i$ each having at least two intersection points with $G$ between $A$ and $B$, say $A_{\lambda}, B_{\lambda}$, as close to one another as needed. By the definition of $G_{i, j}$, at points $A_{\lambda}, B_{\lambda}$, some curves $x_{\mu}^{j}(t)$ and $x_{\eta}^{j}(t)$ have local minima relative to $x_{\lambda}^{i}(t)$. We get a contradiction with the fact that curves in the same family $j$ do not intersect.

Suppose that $G_{i . j}$ is not homeomorphic to $(0,1)$. Then one can find a curve $x_{\alpha}^{i}(t)$ intersecting $G_{i, j}$ more than twice which we proved to be impossible.

\section{An algorithm synthesising finite control strategies}

Without loss of generality let us assume $\ell=2$. Consider the following piecewise integral curve $C$ passing through $\left(t_{0}, x_{0}\right) \in U$. Choose at $\left(t_{0}, x_{0}\right)$ a control $i \in\{1,2\}$ realising the maximal possible speed $\dot{x}_{0}^{i}\left(t_{0}\right)$. There are two possible cases.

1. $\dot{x}_{0}^{1}\left(t_{0}\right) \neq \dot{x}_{0}^{2}\left(t_{0}\right)$ (assume for definiteness that $\dot{x}_{0}^{1}\left(t_{0}\right)>\dot{x}_{0}^{2}\left(t_{0}\right)$ );

2. $\dot{x}_{0}^{1}\left(t_{0}\right)=\dot{x}_{0}^{2}\left(t_{0}\right)$. 
In case (1), for every point $\left(t^{\prime}, x^{\prime}\right)$ on the curve $x_{0}^{1}(t)$ sufficiently close to $\left(t_{0}, x_{0}\right)$, the inequality $\dot{x}_{0}^{1}\left(t^{\prime}\right)>\dot{x}_{\alpha}^{2}\left(t^{\prime}\right)$ holds true for any $x_{\alpha}^{2}(t)$ such that

$$
x_{0}^{1}\left(t^{\prime}\right) \cap x_{\alpha}^{2}\left(t^{\prime}\right)=\left(t^{\prime}, x^{\prime}\right) .
$$

This follows from the transversely of $x_{0}^{1}(t)$ and $x_{0}^{2}(t)$ at $\left(t_{0}, x_{0}\right)$. Therefore, in case (1), at any such point $\left(t^{\prime}, x^{\prime}\right)$ there is no reason to switch from 1 to 2 .

In case $(2)$, there are the following possibilities.

(i) $\left(t_{0}, x_{0}\right)$ is an inflection point of $x_{0}^{2}(t)$ relative to $x_{0}^{1}(t)$. In this case $x_{0}^{2}(t)$ starts above $x_{0}^{1}(t)$ (see Definition 2, (1)), is tangent to $x_{0}^{1}(t)$ at $\left(t_{0}, x_{0}\right)$ and then goes below $x_{0}^{1}(t)$. For any point $\left(t^{\prime}, x^{\prime}\right)$ on $x_{0}^{1}(t)$ sufficiently close to $\left(t_{0}, x_{0}\right)$ there is no reason to switch from 1 to 2 .

(ii) $\left(t_{0}, x_{0}\right)$ is an inflection point of $x_{0}^{1}(t)$ relative to $x_{0}^{2}(t)$. This case is analogous to $(\mathrm{i})$.

(iii) $\left(t_{0}, x_{0}\right)$ is a local minimum point of $x_{0}^{2}(t)$ relative to $x_{0}^{1}(t)$ (see Definition 2, $(3))$. We choose the control $i=2$ in this case.

(iv) $\left(t_{0}, x_{0}\right)$ is a local minimum point of $x_{0}^{1}(t)$ relative to $x_{0}^{2}(t)$. We choose the control $i=1$ in this case.

Assume, for definiteness, that we have either case $(1)$, or $(2, \mathrm{i})$, or (2, iii). According to Lemma 1 , in case (iii) $\left(t_{0}, x_{0}\right)$ is an isolated local minimum point on $x_{0}^{1}(t)$. It follows that the nearest point to $\left(t_{0}, x_{0}\right)$ on $x_{0}^{1}(t)$ at which there may be a reason to switch to the family 2 is the nearest point at which some curve $x_{\alpha}^{2}(t)$ has a local minimum relative to $x_{0}^{1}(t)$. According to Lemma 1 , there is a finite number of such points on $x_{0}^{1}(t)$. Moreover, since these points are definable in the first order theory of the reals, we can bound their number from above via the format of $\gamma$.

\section{Algorithm.}

1. If at $\left(t_{0}, x_{0}\right)$ the integral curves $x_{0}^{i}\left(t_{0}\right), i=1,2$ intersect transversely then choose at $\left(t_{0}, x_{0}\right)$ a control $i \in\{1,2\}$ realising the maximal possible slope $\dot{x}_{0}^{i}\left(t_{0}\right)$. Let it be for definiteness $i=1$.

If $\left(t_{0}, x_{0}\right)$ is an inflection point of the integral curves (for definiteness, let $\left(t_{0}, x_{0}\right)$ be an inflection point of $x_{0}^{2}(t)$ relative to $\left.x_{0}^{1}(t)\right)$ choose the control $i=1$.

If $\left(t_{0}, x_{0}\right)$ is a local minimum of one integral curve relative another (for definiteness, let $x_{0}^{1}(t)$ have local minimum relative to $\left.x_{0}^{2}(t)\right)$ then choose the control $i=1$.

2. The curve $x_{0}^{1}(t)$ has the finite number of local minima relative to it of curves of the type $x_{\alpha}^{2}(t)$. Choose the right-closest to $\left(t_{0}, x_{0}\right)$ on $x_{0}^{1}(t)$ such minimum, and let it correspond to $\alpha=1$. Denote the minimum point by $\left(t_{1}, x_{1}\right)$. Observe that for the smallest $j>0$ for which the $j$ th derivative $x_{1}^{2, j}\left(t_{1}\right)$ of $x_{1}^{2}\left(t_{1}\right)$ is larger than $\dot{x}_{0}^{1}\left(t_{1}\right)=\dot{x}_{1}^{2}\left(t_{1}\right)$ we have $x_{1}^{2, j}\left(t_{1}\right)>x_{0}^{1, j}\left(t_{1}\right)$.

3. The curve $x_{1}^{2}(t)$ has the finite number of local minima relative to it of curves of the type $x_{\beta}^{1}(t)$. Choose the right-closest to $\left(t_{1}, x_{1}\right)$ on $x_{1}^{2}(t)$ such minimum, and let it correspond to $\beta=2$. Denote the minimum point by $\left(t_{2}, x_{2}\right)$. 
Observe that for the smallest $j>0$ for which the $j$ th derivative $x_{2}^{1, j}\left(t_{2}\right)$ of $x_{2}^{1}\left(t_{2}\right)$ is larger than $\dot{x}_{1}^{2}\left(t_{2}\right)=\dot{x}_{2}^{1}\left(t_{2}\right)$ we have $x_{2}^{1, j}\left(t_{2}\right)>x_{1}^{2, j}\left(t_{2}\right)$.

4. Continue recursively until at a point $\left(t_{k}, x_{k}\right)$ a control $i_{k} \in\{1,2\}$ will be chosen realising the local minimum of some $x_{k}^{i_{k}}(t)$ relative $x_{k-1}^{i_{k-1}}(t)$, and there will be no right-closest tangent points of curves from the complement family. If $x_{k}^{i_{k}}(t)=\omega$ for some $t_{k}<t \leq 1$ then $\omega$ is reachable, otherwise not.

Theorem 1. The number of steps in the Algorithm is finite, moreover, in algebraic case it is bounded from above via the format of $\gamma$.

Proof. Let $\left(t_{\ell}, x_{\ell}\right)$ be a switching point of the piecewise integral curve $C$ constructed in the Algorithm, for definiteness from $i_{\ell-1}=1$ to $i_{\ell}=2$. Let $G$ be a connected component of $G_{1,2}$ such that $\left(t_{\ell}, x_{\ell}\right) \in G$. According to Lemma 2, $\left(t_{\ell}, x_{\ell}\right)$ is the unique point of intersection of $G$ and $x_{\ell}^{i_{\ell}}(t)$. Recall from the beginning of Section 3 that the integral curve $x_{\ell}^{i_{\ell}}(t)$ divides $U$ into the upper part $U_{+}\left(i_{\ell}, \ell\right)$ and the lower part $U_{-}\left(i_{\ell}, \ell\right)$.

If $G \cap U_{+}\left(i_{\ell}, \ell\right)=\emptyset$ then there will be no point $\left(t_{m}, x_{m}\right) \in C$ with $\ell<m$ such that $\left(t_{m}, x_{m}\right) \in G$. So, suppose that $G_{+}:=G \cap U_{+}\left(i_{\ell}, \ell\right)$ is non-empty. Since, by Lemma $2, G$ is homeomorphic to $(0,1)$, so is $G_{+}$. One endpoint of $G_{+}$is $\left(t_{\ell}, x_{\ell}\right)$, while for the other endpoint, denoted by $A$, we have three possibilities:

(i) $A \in\{t=-1, x<1\}$, i.e., $A$ belongs to the left-hand vertical side of the square $U$

(ii) $A \in\{x=1\}$, i.e., $A$ belongs to the upper side of $U$;

(iii) $A \in\{t=1\}$, i.e., $A$ belongs to the right-hand vertical side of the $U$.

In any case $G_{+}$divides $U_{+}\left(i_{\ell}, \ell\right)$ into two domains. In case (i) the left domain is the one which is adjacent to $\{t=-1\}$ and not adjacent to $\{x=1\}$, the other is the right domain. In case (ii) the left domain is adjacent to the left interval into which $A$ divides $\{x=1\}$, the other is the right domain. In case (iii) the right domain is the one which is adjacent to $\{t=1\}$ and not adjacent to $\{x=1\}$, the other is the left domain.

Note that the point $\left(t_{\ell+1}, x_{\ell+1}\right) \in C$ belongs to the right domain otherwise $x_{\ell}^{i_{\ell}}(t)$ would have at least two intersection points with $G$ which is impossible by Lemma 2.

Suppose there is a switching point $\left(t_{m}, x_{m}\right) \in C$ with $\ell<m$ such that $\left(t_{m}, x_{m}\right) \in G_{+}$, and let this be the first such point between $\ell$ and $m$. The curve $x_{m-1}^{i_{m-1}}(t)$ "approaches $G_{+}$from the right" (this means that $x_{m-1}^{i_{m-1}}(t)$, restricted to $\left[t_{m-1}, t_{m}\right]$, lies in the right domain). There are two possibilities: either $x_{m-1}^{i_{m-1}}(t)$ is "tangent" to $G_{+}$(i.e., after passing $\left(t_{m}, x_{m}\right)$ remains in the right domain) or enters the left domain. In case the first possibility realises, $G_{+}$remains under the graph $x_{m-1}^{i_{m-1}}(t)$ and never appears again.

Consider the second possibility. Suppose we have case (i) (see above). If the origin of the integral curve $x_{m-1}^{i_{m-1}}(t)$ belongs to the right domain, then after entering the left domain at $\left(t_{m}, x_{m}\right)$ it has to leave the left domain at some moment $t^{\prime}>t_{m}$ in order to reach either $\{t=1\}$ or $\{x=1\}$. This contradicts to 
the uniqueness of the intersection point $G \cap x_{m-1}^{i_{m-1}}(t)$. If the origin of $x_{m-1}^{i_{m-1}}(t)$ belongs to the left domain, then $x_{m-1}^{i_{m-1}}(t)$ already intersected $G$ at some moment $t^{\prime \prime}<t_{m}$ in order to reach the right domain, and we have a contradiction again.

In cases (ii) or (iii), the curve $x_{m-1}^{i_{m-1}}(t)$ always enters the right domain by crossing $G_{+}$(rather than $\left.x_{\ell}^{i_{\ell}}(t)\right)$. Indeed, $x_{\ell+1}^{i_{\ell+1}}(t)$ has a local minimum at the point $\left(x_{\ell+1}, t_{\ell+1}\right)$ and is the closest to $\left(t_{\ell}, x_{\ell}\right)$ with this property, and therefore $x_{\ell+1}^{i_{\ell+1}}(t)$ enters the right domain by crossing $G_{+}$. But $x_{m-1}^{i_{m-1}}(t)$ is either above $x_{\ell}^{i_{\ell}}(t)$ or above $x_{\ell+1}^{i_{\ell+1}}(t)$. So we get the same contradiction as before.

We conclude that $G$ is intersected by $C$ at most twice. Since the switching points of $C$ belong to the connected components $G$ the number of these points does not exceed twice the number of the connected components. The latter is finite, and in algebraic case estimated from above via the format of $\gamma$.

Theorem 2. Let the polynomials $P$ defining Nash functions $\Gamma^{i}$ have degrees not exceeding $d$. Then for the piecewise integral curve $C$, constructed in the Algorithm, $k=d^{O(1)}$.

Proof. It follows from the proof of Theorem 1 that it is sufficient to estimate the number of connected components of the curve $G_{1,2}$. This curve is definable by a formula (with quantifiers) of the first order theory of the reals with some fixed finite number of variables. According to [4], the number of connected components of $G_{1,2}$ does not exceed $k=d^{O(1)}$.

Definition 4. A piecewise integral curve $C_{\mathrm{opt}}$ reaching $\Omega$ from $\left(x_{0}, t_{0}\right)$ at the moment $t_{\mathrm{opt}}$ is called time-optimal if $t_{k}^{\mathrm{opt}} \leq t_{k}$ for any $C$ reaching $\Omega$ from $\left(x_{0}, t_{0}\right)$ at some moment $t_{k}$. If $t_{k}^{\mathrm{opt}}<t_{k}$, we say that $C_{\mathrm{opt}}$ is faster than $C$.

Theorem 3. The piecewise integral curve C, constructed in the Algorithm, is time-optimal. In particular, the Algorithm is correct.

Proof. Let

$$
C=\bigcup_{0 \leq j \leq k-1}\left\{(t, x) \in U \mid t \in\left[t_{j}, t_{j+1}\right], x=x_{j}^{i_{j}}(t)\right\}
$$

We prove the theorem by induction on $k$.

If $k=1$ (the base of the induction), then $C$ is the integral curve $x_{0}^{i}(t)$ restricted on $\left[t_{0}, t_{1}\right]$, i.e., $C=\left\{(t, x) \in U \mid\left[t_{0}, t_{1}\right], x=x_{0}^{i}(t)\right\}$, and there are no other integral curve $x_{\alpha}^{j}(t)$ and a point $\left(t^{\prime}, x^{\prime}\right) \in C$, such that $j \neq i$ and $x_{\alpha}^{j}(t)$ has a local minimum relative to $x_{0}^{i}(t)$ at $\left(t^{\prime}, x^{\prime}\right)$. If $C$ was not time-optimal then there would be at least one integral curve $x_{\beta}^{j}(t)$ whose restriction on a subinterval $\left[t^{\prime}, t^{\prime \prime}\right]$ of $\left[t_{0}, t_{1}\right]$ included in a faster piecewise integral curve. Then there would be an integral curve $x_{\alpha}^{j}(t)$ in the family $j$ (possibly, with $\alpha=\beta$ ) having a local minimum relative to $x_{0}^{i}(t)$ at some $\left(t^{\prime}, x^{\prime}\right) \in C$. We got a contradiction.

Let $k>1$. Suppose there is a faster than $C$ piecewise integral curve $C^{\prime}$. Let $x_{1}:=x_{1}^{i_{1}}\left(t_{1}\right)$. The line $\left\{(t, x) \mid x=x_{1}\right\}$ is reached by $C^{\prime}$ at some moment $t^{\prime}$. If 
$t^{\prime}<t_{1}$ then we get the case as in the base of the induction (replacing $\omega$ by $x_{1}$ ) which we led to a contradiction.

If $t^{\prime}=t$ then by the inductive hypothesis the piecewise integral curve $C$ restricted on $\left[t_{1}, t_{k}\right]$ is time-optimal for the initial point $\left(t_{1}, x_{1}\right)$, hence $C^{\prime}$ is not faster than $C$ and we get a contradiction.

If $t^{\prime}>t$ then, since $C^{\prime}$ is faster than $C$, there exists a point $\left(t^{\prime \prime}, x^{\prime \prime}\right) \in C \cap C^{\prime}$, where $t^{\prime \prime}>t^{\prime}$, and by the inductive hypothesis $C$ restricted on $\left[t^{\prime \prime}, t_{k}\right]$ is timeoptimal for the initial point $\left(t^{\prime \prime}, x^{\prime \prime}\right)$. It follows that $C^{\prime}$ is not faster than $C$ and we get a contradiction.

Remark 3. The number $t_{k}$ in the piecewise integral curve, computed by the Algorithm, is the minimal time required to reach $\Omega$ from the initial point $\left(t_{0}, x_{0}\right)$. Thus, the Algorithm solves the optimal control problem of finding a time minimization strategy.

\section{Conclusion, related, and future work}

O-minimality is an fruitful theoretical concept for formal verification of dynamical and hybrid systems. It has been successfully used for proving that certain classes of dynamical and hybrid systems admit finite-state bisimilar models [10, $5,8]$; for computing combinatorial types of trajectories [9]; for mode switching synthesis [7], etc. In this paper we investigated synthesis of finite control strategy for one-dimentional controlled polynomial dynamical systems while setting up the framework for synthesis of finite control strategies for wider classes of controlled dynamical systems and studying related problems.

The main interesting future development would be to consider the domains of integral curves to be more general than a rectangle, and possibly to include critical values of the projection $\pi$. Of course, generalizations of the problem to higher dimensions are of great interest.

Finally, let us mention a two-person zero-sum game version of our problem. In a game the right of the first move is prescribed. When it's a player's turn to move, at a moment $t_{i}$, it chooses a control $y_{i+1} \in \gamma^{-1}(x) \cap\left\{t=t_{i}\right\}$ and a stopping time $t_{i+1}$. At the moment $t_{i+1}$ the other player makes a move. The player 1 wins (player 2 looses) if and only if $\Omega$ is reached. In the Example 1 , let $\omega>\alpha^{2}$. The player 1 always looses, independently of who is prescribed to make the first move if $x_{0}<\alpha^{2}+\alpha t_{0}^{2}$. If $x_{0} \geq \alpha^{2}+\alpha t_{0}^{2}$ then the player 1 wins if and only if it has the first move, or it has the second move but $x_{0} \geq \omega-\sqrt{\omega} t_{0}^{2}$. It would be interesting to construct a general efficient algorithm for computing optimal strategies in dynamical games from this class.

\section{References}

1. Eugene Asarin, Oded Maler, and Amir Pnueli, Reachability analysis of dynamical systems having piecewise-constant derivatives, Theoret. Comput. Sci. 138, N 1, pp. $35-65$. 
2. Eugene Asarin, Venkatesh Mysore, Amir Pnueli, and Gerardo Schneider, Low dimensional Hybrid Systems - Decidable, Undecidable, Don't Know, Accepted for publication in Information and Computation, 2010.

3. Jacek Bochnak, Michel Coste, Marie-Franoise Roy, Real Algebraic Geometry, [Results in Mathematics and Related Areas (3)], 36. Springer-Verlag, Berlin, 1998

4. Saugata Basu,Richard Pollack, Marie-Franoise Roy, Algorithms in real algebraic geometry, Second edition. Algorithms and Computation in Mathematics, 10. Springer-Verlag, Berlin, 2006. $\mathrm{x}+662 \mathrm{pp}$.

5. T. Brihaye and C. Michaux, On the expressiveness and decidability of o-minimal hybrid systems, Journal of complexity 21 (4), 2005, pp. 447-478.

6. Thomas Brihaye, A note on the undecidability of the reachability problem for ominimal dynamical systems, Math. Log. Q., v. 52, N 2, 2006, pp. 165-170.

7. Patricia Bouyer, Thomas Brihaye and Fabrice Chevalier, Control in o-minimal Hybrid Systems, In Proc. LICS'06, 2006, pp.367-378.

8. Margarita Korovina and Nicolai Vorobjov, Pfaffian Hybrid Systems, In Proc. CSL'04, LNCS 3210, 2004, pp. 430-441.

9. Margarita Korovina and Nicolai Vorobjov, Computing combinatorial types of trajectories in Pfaffian Dynamics, Journal of Logic and Algebraic Programming, 79 (1), 2010, pp. 32-37.

10. G. Lafferriere, G. J. Pappas, and S. Sastry, O-minimal hybrid systems, Math. Control Signals Systems 13 (1), 2000, pp. 1-21. 